A general method for the design and fabrication of shape memory alloy active spring actuators

This article has been downloaded from IOPscience. Please scroll down to see the full text article.

2012 Smart Mater. Struct. 21115029

(http://iopscience.iop.org/0964-1726/21/11/115029)

View the table of contents for this issue, or go to the journal homepage for more

Download details:

IP Address: 193.205.81.18

The article was downloaded on 08/11/2012 at 10:50

Please note that terms and conditions apply. 


\title{
A general method for the design and fabrication of shape memory alloy active spring actuators
}

\author{
M Follador ${ }^{1,2}$, M Cianchetti $^{1}$, A Arienti $^{1}$ and C $^{\text {Laschi }}{ }^{1}$ \\ ${ }^{1}$ The BioRobotics Institute, Scuola Superiore Sant'Anna, Pisa, Italy \\ ${ }^{2}$ Centre for Micro-BioRobotics of Italian Institute of Technology @SSSA, Pontedera, Pisa, Italy \\ E-mail: m.follador@sssup.it
}

Received 11 May 2012, in final form 6 August 2012

Published 23 October 2012

Online at stacks.iop.org/SMS/21/115029

\begin{abstract}
Shape memory alloys have been widely proposed as actuators, in fields such as robotics, biomimetics and microsystems: in particular spring actuators are the most widely used, due to their simplicity of fabrication. The aim of this paper is to provide a general model and the techniques for fabricating SMA spring actuators. All the steps of the design process are described: a mechanical model to optimize the mechanical characteristic for a given requirement of force and available space, and a thermal model for the estimation of the electrical power needed for activation. The parameters of both models are obtained by experimental measurements, which are described in the paper. The models are then validated on springs manufactured manually, showing also the fabrication process. The design method is valid for the dimensioning of SMA springs, independently from the external ambient conditions. The influence on the actuator bandwidth was investigated for different working environments, providing numerical indications for the utilization in underwater applications. The spring characteristics can be calculated by the mechanical model with an accuracy of $5 \%$. The thermal model allows one to calculate the current needed for activation under different ambient conditions, in order to guarantee activation in the specific loading conditions. Moreover, two solutions were found to reduce the power consumption by more than $40 \%$ without a dramatic reduction of bandwidth.
\end{abstract}

(Some figures may appear in colour only in the online journal)

\section{Introduction}

Shape memory alloys (SMAs) have been proposed in robotics as an alternative to conventional electric, hydraulic and pneumatic motors, because of the increasing need to develop smaller and lighter robots. Indeed, one of the advantages of this technology is that the lighter the actuators, the bigger is the resulting power/weight ratio, therefore making SMAs a valid alternative to conventional actuators (figure 1) [1-3].

Another consideration that makes SMAs a valid alternative to traditional actuation is that they can work in a liquid environment without losing their mechanical properties. The principal application is found in the propulsion system of bioinspired robots, using the alloy in the form of wires [4-6] or in the form of springs [7]. The approach used in the design of underwater actuators is generally the same as in the design of actuators that work in air. The heat exchange problem was considered, from a control point of view, as a fixed parameter to take into account in the model [6] or in the simulations [4], and derived analytically or experimentally for the specific case and the specific application. Further advantages of using SMAs are the relatively low cost and the large number of methods that can be employed in the manufacturing process to obtain an actuator $[8,9]$. The simplest way is to use the alloy in the form of wires, which present a very high force density, but that have the disadvantages of a limited stroke (maximum 
$8 \%$ ) and the need for a high recovery force. Wires can be shaped as springs, to obtain actuators capable of performances that are the result of a trade off between maximum stroke and force, which depend only on geometrical factors. SMA springs have multiple applications, including the actuation of rigid joints [10, 11], providing bending motion in flexible structures [12], or fabrication of micropumps [13]. Moreover, thanks to all the above-mentioned peculiarities, they are good candidates for biomimetic and bioinsipired robots, where light weight, small dimensions and low stiffness are fundamental.

There are three main issues connected to the design and fabrication of SMA springs: (1) modelling the mechanical performances of the spring, (2) modelling the thermodynamic cycles during heating and cooling, and (3) dimensioning the control current. Often these issues are approached as a single problem of controlling the alloy using a thermo-mechanical model as feedback for a closed loop control [14, 15].

Many authors have dealt with the first issue and found different solutions [16, 17], starting from the knowledge derived from considerations of phase transformation in the single crystals. This approach is purely mathematical and takes into consideration many parameters, thus obtaining very general models. A simpler way to approach the problem is to use a constitutive model based on the classical equations for linear-elastic springs with variable material parameters in order to take into account the phase transition and hysteresis [18]. In this paper, mechanical characteristics of the SMA spring are described by a simple linear-elastic model, with parameters depending on the crystalline phase.

The second issue has been investigated in the literature in different ways, with the aim of finding a complete model of the heating and cooling cycles based on both analytical formulation and experimental evidence, in order to predict the thermodynamic behaviour of the alloy under specific conditions [19, 20]. The method proposed here, on the other hand, is based on a simple but robust semi-empirical procedure for the estimation of the thermal exchange with the environment and at the same time for the estimation of the electric power necessary for the activation.

Finally, the dimensioning control current, induced by a PWM, is a direct consequence of the thermodynamics investigation.

Shape memory alloy can be manufactured in many different shapes, providing diverse means of actuation depending on the geometry adopted for the specific application. Among all the possible actuation solutions, the helical shape is one of the most frequently used, due to the number of parameters that can be adjusted, its ease of fabrication and its compactness. Consequently, the aim of this paper is to show the design method for the realization of a SMA spring actuator for a generic application, considering the most common design parameters, i.e. stroke and force. Further considerations were made on the working environment of the actuators, measuring the heat exchange in numerous conditions. Different springs were realized, following these indications, to validate the single models. The design method, here proposed and validated, found application in the realization of springs used as actuators for a soft manipulator

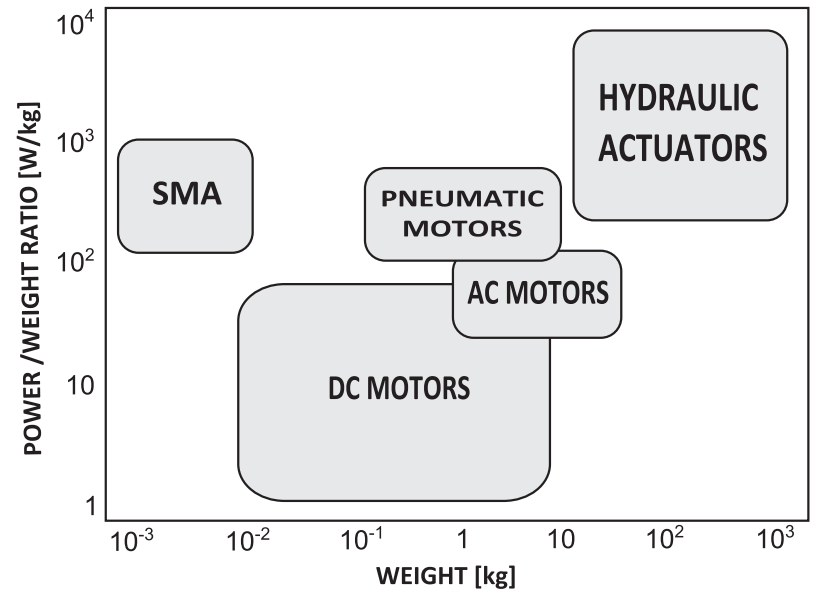

Figure 1. Adapted from [3]. Diagram showing the power/weight ratio versus weight of different actuation technologies. Low-weight SMAs show better performances with respect to traditional actuators.

inspired by an octopus arm, providing bending, elongation and shortening mechanisms [21, 22]. The robotic arm has, in the internal part, a hollow cavity, where the actuators are positioned, filled with a liquid medium that equilibrates the external pressure. The composition of the liquid medium is optimized to find a trade off between the actuator frequency bandwidth and power consumption. Necessary specifications for this application are light weight, reduced dimensions, fast activation and reliability. A position control is not necessary, because the control of the spring activation is just on-off. SMA springs are a good solution for the implementation of the transversal and longitudinal actuators, because they do not influence significantly the stiffness of the arm, are small enough to be integrated inside the arm, can work in a water environment and can exert enough force to provide the elongation and shortening of the arm.

A simplified approach to the use of SMA technology and methods to reduce power consumption without sacrificing the performances of the actuators are analysed. Thus, in the first part of the paper, a description of the design of a SMA spring actuator is presented, starting from the requirements of dimensions, stroke and force, applying sequentially the steps of a mechanical model and a thermal model based on an experimental procedure. In the second part, both models are validated, and a method for the manual fabrication is also shown. Finally, the effect of the ambient conditions on the performance of the spring is discussed. The steps of the manual fabrication provide the indications of an easy, fast and low-cost solution to obtain the desired springs to be tested in the early phases of the design of a generic actuation system. The mechanical and thermal models are the tools for the dimensioning of the actuators, and are valid also to define characteristics to search for among commercially available SMA springs or to set customized values to request for. The springs fabricated and tested are used only for the validation process and represent a generic case study. 


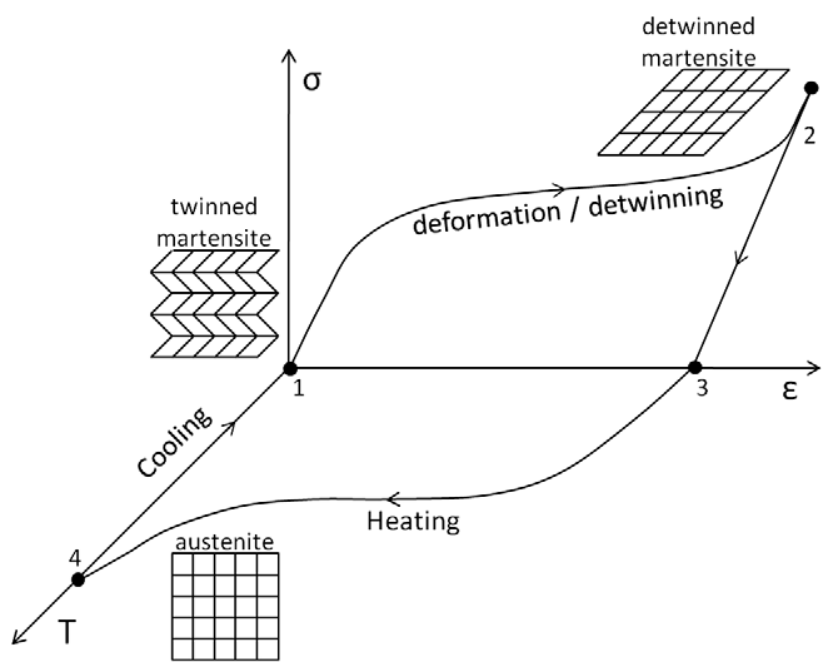

Figure 2. Schematic representation of shape memory effect. At point (1) the alloy is in the twinned martensitic phase; when load is applied (1-2) the crystal configuration changes from twinned to detwinned, and the deformation remains also when the load is removed (2-3). Increasing the temperature results in a change into the austenitic phase, with a consequent recovery of the original shape (3-4), which is maintained also when the alloy cools down (4-1).

\section{SMA theory}

The shape memory effect was first discovered in the 1960s [23] on a nickel-titanium alloy, and since that time it has been an important subject of research for its interesting properties. The macroscopic effect that is exploited for the actuation process is the recovery of a pre-determined geometrical shape, after a plastic deformation, triggered and completed by a heating process (figure 2). This effect is based on the change of the crystalline phase from martensitic, which is thermodynamically stable at lower temperature, to austenitic, which is stable at higher temperature.

The phase transition occurs in a certain range of temperature and exhibits a high hysteresis, so it is necessary to define four temperatures that characterize quantitatively the effect: $A_{\mathrm{s}}, A_{\mathrm{f}}, M_{\mathrm{s}}$, and $M_{\mathrm{f}}$. These are the temperatures that respectively correspond to the start and finish of the austenitic transformation during heating process $\left(A_{\mathrm{s}}, A_{\mathrm{f}}\right)$ and of the martensitic transformation during cooling process $\left(M_{\mathrm{s}}, M_{\mathrm{f}}\right)$. These temperatures depend also on the internal stress of the alloy: there is a linear correlation between stress and transition temperatures. A thorough review of the basic properties and the constitutive behaviour of the SMAs can be found in [24].

\section{Models definition}

The SMA actuator, for our purpose, is considered as a spring with two different spring rates, depending only on the crystalline phase of the alloy. Since in our assumption the actuator is used just in an on-off configuration, intermediate situations where the two phases coexist are neglected. Spring contraction is considered to occur when a certain activation temperature is reached, while elongation is provided by an antagonistic mechanism that involves another SMA spring.

The design of the SMA helical springs has three geometrical parameters to consider:

- Wire diameter

- Average spring diameter

- Number of coils.

A mechanical model is used to describe the mechanical characteristics of the spring and will be shown in section 3.2. The three parameters are the output of a simple algorithm developed in MATLAB (MathWorks, Inc.) that calculates the admissible solutions that match the specified requirements of force and available workspace. Thus the algorithm inputs are the mechanical characteristics of the alloy, the target force and the available space to lodge it, while wire diameter, spring diameter and number of coils are considered as variables of the algorithm for the choice of the spring. The spring that fits the specifications with the minimum power consumption is considered as the best solution. Subsequent to the mechanical model, a thermal analysis of the wire is the next step to control the spring activation. A thermal model is used to predict the temperature reached by the wire for a given electric power, so that the spring can be activated while avoiding overheating. Some other solutions, described in section 5.1, are used to avoid excessive internal stress during working cycles.

In our specific case the choice of the raw material for the springs was directed towards a commercially available alloy (Smartflex(C) SAES getters group, Milano, Italy).

\subsection{Material characterization}

The chosen wires have a nominal activation temperature $A_{\mathrm{s}}$ of $55^{\circ} \mathrm{C}$ and $A_{\mathrm{f}}$ of $60^{\circ} \mathrm{C}$. The first data necessary to develop the model are the Young's moduli of both the martensitic and the austenitic phases.

In our case, the mechanical properties were not indicated on the data sheet, so they were investigated by a material testing device (INSTRON 4464, Norwood, MA) on wires of two different diameter sizes: 100 and $200 \mu \mathrm{m}$. A series of tests was performed for each diameter with a tensile speed of $0.1 \mathrm{~mm} \mathrm{~s}^{-1}$ until the break of the specimen. The Young's modulus was extracted for each trial, from which the mean values were calculated. The Young modulus was found to be $22 \mathrm{MPa}$ for the martensitic phase and $55 \mathrm{MPa}$ for the austenitic phase.

Tests on the austenitic phase were performed by connecting a stabilized power supply directly to the wire, once logged on the tensile machine. Current was driven into the wire to reach the activation temperature and maintained for the duration of the test. Different current values were tested to check repeatability.

\subsection{Mechanical model}

A mechanical model is a necessary tool for the prediction of the performance of the springs, and the aim of this work is to 
design an actuator starting only from force requirements and available space. The model is therefore subdivided into two parts: the first one calculates the actuator performance and the second one iteratively searches for the optimized solution for the proposed case.

In the first part, the variable input data for the model are: wire diameter; spring index (ratio between average spring diameter and wire diameter) and number of coils. Poisson's ratio, maximum internal stress and Young modulus (austenitic and martensitic) are the material fixed parameters. The model is based on Castigliano's theorem and gives the spring rate (for austenite and martensite) and maximum force as output. The maximum internal stress of the spring is coincident with the maximum shear stress $\left(\tau_{a}\right)$ : to have a fatigue life of more than 100000 cycles, its value must be $\tau_{a}<450 \mathrm{MPa}$, according to manufacturer's data sheet. For design purposes, the maximum stress is set to $\tau_{a}=450 \mathrm{MPa}$. The inputs of the model are then: wire diameter $(d)$, spring index $(c)$, number of coils $(n)$, Poisson's ratio $(v)$, Young modulus $(E)$, which has to be adjusted when considering the martensitic or austenitic phase.

The shear modulus can be calculated as:

$$
G_{a}=\frac{E}{2(1+v)}(\mathrm{MPa}) .
$$

The spring rate $(K)$ can be derived as:

$$
K=\frac{G_{a} d^{4}}{8 n D^{3}}\left(\mathrm{~N} \mathrm{~mm}^{-1}\right) .
$$

Equations (1) and (2) have to be calculated for both austenitic and martensitic phases, using the appropriate terms.

The maximum force that can be exerted by the spring can be calculated as:

$$
F=\frac{\tau_{a} \pi d^{3}}{8 D}(\mathrm{~N})
$$

After the development of this first part of the model and its validation (shown in section 4.2), the second step, consisting of iterative optimization, was implemented in MATLAB. In this second part, two additional inputs are inserted into the algorithm to take into account the available space for the working cycles of the springs and the minimum force that the springs have to exert during the working cycles. The input parameters are expressed in the form of vectors and all possible combinations are calculated. All results are stored, but only the ones that satisfy the requirements of feasibility, stroke and force are considered. Then the results are sorted according to the desired sequence (stroke, force, power consumption) in order to give the user the possibility to visually check and choose the most suitable result. With these two additional inputs it is possible to choose an actuator that can be used in a defined space and can achieve the minimum required force. From the geometrical parameters it is then possible to compute the electrical parameters (resistance and current needed for phase transition) to estimate the power needed for activation. Moreover from the characteristics of the resulting spring, a bias spring can be dimensioned to work as antagonistic actuator.

\subsection{Thermal model}

The thermodynamics of the heating and cooling cycles is a critical point in the design, both for the power consumption and for the frequency of the activation cycles. In particular, the heat transfer coefficient relates directly to the temperature reached at steady state and electric power provided during activation. It is not easy to derive it analytically and it is not known a priori; thus a model is needed to calculate it from simple measurements. A test protocol was developed for this purpose. A single equation allows one to calculate the heat transfer coefficient, having as input a steady state temperature and the corresponding current needed to reach it. In the proposed method the two data are collected in separate experiments: in the first, the transformation temperature is measured, and in the second the current needed to obtain a phase transformation is evaluated through the resistivity variation of the wire due to phase transformation. From now on, this procedure will be called the indirect method. The same result of estimation of the heat transfer coefficient could be obtained by measuring the power supplied to the wire and the temperature directly on the surface of the wire at steady state, by means of a thermocouple. In this way the two data are collected at the same time, and some measurement errors can be reduced, but, since the use of a very small thermocouple is limited to measuring temperatures in air on wires with a diameter at least of the same size of the thermocouple, this direct method is used just to validate the indirect method. On the other hand, the indirect method can be utilized to estimate the heat transfer coefficient on all wire sizes and in different ambient conditions. Tests on the same wire led to similar results of estimation of the heat transfer coefficient (see section 4.3). A detailed description of each step will be shown in the next sections.

3.3.1. Heat transfer coefficient calculation. In our case, heat transfer is dominated by convection, since the wire is surrounded by low viscosity fluids (air, water and glycerol-water solutions). Radiation is neglected because of the relatively low temperatures of the surfaces. Hence, heat transfer is determined by the heat transfer coefficient $(h)$. Within our temperature range, this coefficient is considered independent of temperature, as found in [19], and the heat transfer is expressed by the Fourier law, which for steady state problems is:

$$
q=h A\left(T-T_{a}\right)
$$

where $q$ is the thermal power generated, $A$ is the heat exchange surface area, $T a$ is the external temperature and $T$ is the temperature on the surface of the wire.

Coefficient $h$ can be calculated from a specific thermal power and the corresponding steady state temperature [20]. Equation (4) can be rewritten to express the coefficient as function of the electric current as:

$$
h=\frac{I^{2} \rho}{2 \pi^{2} r^{3}\left(T-T_{a}\right)}
$$

where $\rho$ is the resistivity and $r$ the radius of the wire. 
Given the target temperature, which in our case is the activation temperature, the external temperature of the fluid and the electric current, it is then possible to calculate the heat transfer coefficient.

3.3.2. Activation temperature measurement. The activation temperature can be estimated by measuring the temperature-contraction relationship of the wire. An experimental setup was built for this purpose. A $200 \mathrm{~mm}$ SMA wire (diameter $0.2 \mathrm{~mm}$ ) was manually stretched to reach a $5 \%$ elongation in its central part (avoiding elongation at the extremities). The wire was then fixed on one end to a wooden block by an aluminum plate and screws.

The other end was fixed on a slide potentiometer (Panasonic, $10 \mathrm{k} \Omega$, operating force $\max 1 \mathrm{~N}$ ). The voltage-position relation was previously measured to use it as a position sensor. A type $K$ calibrated thermocouple was fixed in proximity to the wire to measure the ambient temperature. The thermocouple and potentiometer were connected to a data acquisition board (SCB68-National Instruments), then the temperature and displacement were recorded with LabView (National Instruments). The entire system was then positioned inside a programmable oven (VD 53-Binder) and the temperature was raised until the complete activation was reached. The temperature at complete activation was found to be $63{ }^{\circ} \mathrm{C}$. Errors in this measurement were introduced by the thermocouple, the accuracy of which is estimated to be $\pm 2{ }^{\circ} \mathrm{C}$.

The austenitic transformation temperature $\left(A_{\mathrm{f}}\right)$ depends on the stress condition of the alloy through the following relationship:

$$
\Delta A_{\mathrm{f}}=\frac{\tau}{C_{a}}
$$

where $C_{a}$ is a coefficient that relates the transformation temperature and the internal stress and $\tau$ is the maximum internal stress inside the alloy for a given elongation of the spring. For the alloy the value used was $C_{a}=8.2 \mathrm{MPa}{ }^{\circ} \mathrm{C}^{-1}$ (from materials data sheet). For a given spring geometry and maximum force applied to the spring, the maximum internal stress can be calculated, i.e. the correct activation temperature can be obtained. When calculating the power needed for activation, the target force of the actuator must be used to calculate the actual transformation temperature, which has to be taken as the target temperature for the activation.

3.3.3. Resistivity measurement. The phase-related resistivity was exploited in order to find the current needed to obtain a full phase change. In changing from the martensitic to the austenitic phase, the resistivity decreases. In the transition phase, the resistivity is proportional to the phase ratio present in the wire. By measuring the curve that relates the wire resistance and the current passing in the wire, it is possible to identify the complete transition point, where full austenitic transformation occurs, which is achieved at a known temperature. A PWM control board is used to activate the wire. The resistance is measured indirectly by the ratio of voltage and current. Both are directly measured with the

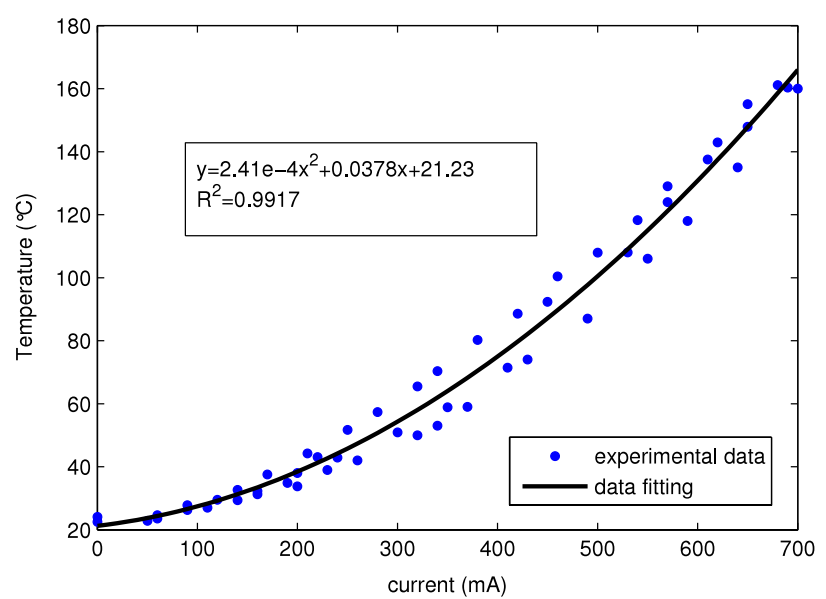

Figure 3. Curve of the temperature at steady state versus current, for a wire in still air.

acquisition board, the first in parallel with the wire, whereas the second is measured by an ammeter.

The resistance is recorded and the difference between the initial value and the steady state value is considered. In the activation temperature range the resistance varies almost linearly. The resistance does not vary prior to the beginning of activation, but once the activation is completed the maximum difference can be observed. The current corresponding to the peak value is considered as the one needed to achieve full phase transformation. This test was also performed in water and in different solutions of glycerol, with the same experimental setup used for air. SMA connection wires were dipped into a Plexiglas box filled with liquid. The volume of the water was sufficient to avoid global heating of the liquid due to the thermal power from the wire, thus a constant ambient temperature can be assumed.

3.3.4. Heat transfer coefficient direct measurement. The heat transfer coefficient was firstly measured using the direct method, following a procedure that consists in (i) measuring the temperature of the surface of the wire and (ii) relating the temperature to the electric power supplied. Measuring the temperature on a $0.2 \mathrm{~mm}$ diameter wire is a complex task because contactless methods, such as laser or thermocameras, have insufficient resolution to focus only on the wire while avoiding the surrounding environment. In addition, thermocouples and thermoresistances can influence the measurement if the diameter of the sensor is comparable to the size of the wire. The solution found to this problem was the use of a thin wire thermocouple (diameter $0.0025 \mathrm{~mm}$ ), with the sensing tip wrapped around the wire. Temperature values were recorded by a data acquisition board and the wire was directly connected to a power supply providing the constant current. The wire was maintained in still air by a surrounding Plexiglas box. The wire was heated until a stable temperature value was recorded. The following activation was performed once the initial temperature was again reached Three trials were carried out and the values interpolated with a second-order polynomial (figure 3). 


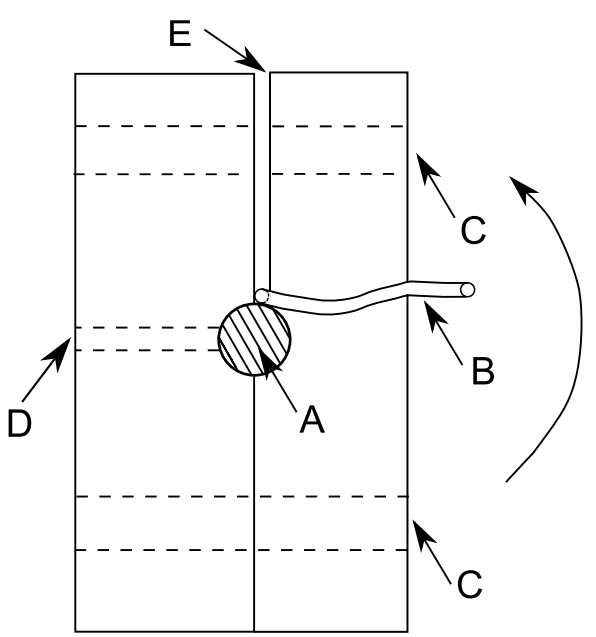

Figure 4. Aluminum device for winding the SMA wire around the steel core. A-steel core; B-SMA wire; C-holes for screw and nuts for fixing the two halves of the block; D-threaded hole for fixing the aluminum block on the steel bar; E—slot for the SMA wire.

\section{Models validation}

\subsection{Manual fabrication of a prototype of SMA spring}

Manual fabrication of the SMA spring is a fundamental step in the design process to realize a large number of prototypes in a relatively fast way at low cost, before eventually choosing commercially available springs or having them fabricated by industrial manufacturers. A good fabrication method is, therefore, necessary to obtain repeatability among different actuator prototypes, since small differences and imprecision can greatly affect the mechanical performance of the springs. To memorize the desired shape, the spring has to be mechanically constrained and maintained at $450{ }^{\circ} \mathrm{C}$ for 30 min inside a furnace. After this time has elapsed, the spring is water quenched. This protocol has been established after a series of experimental trials, also based on indications found in the literature, testing the different fabricated artefacts and choosing the method that produced springs with performances that were more stable and repeatable.

A new method for manual fabrication is proposed, involving the use of two aluminum elements to coil up the wire around the central steel bar. One extremity of the wire is blocked on the core between two halves of an aluminum block that is held tight by two screws. The wire is wound on the core using a simple aluminum device made of two halves, as illustrated in figure 4. The steel bar is held in place with two screws and nuts (holes $\mathrm{C}$ ) between the two parts of the block, and lodged in the central hole (A) whose dimension allows the rotation of the aluminum block. Then the wire (B) is passed in the space between the two parts of the block $(\mathrm{E})$, close to the steel bar. The rotation of the block makes the wire wind around the core, generating the coils of the spring. When the process is complete, an additional screw is used to avoid uncoiling of the spring (hole D).

Figure 5 shows a spring ready to be put in the furnace. The core and aluminum blocks are customized for the specific

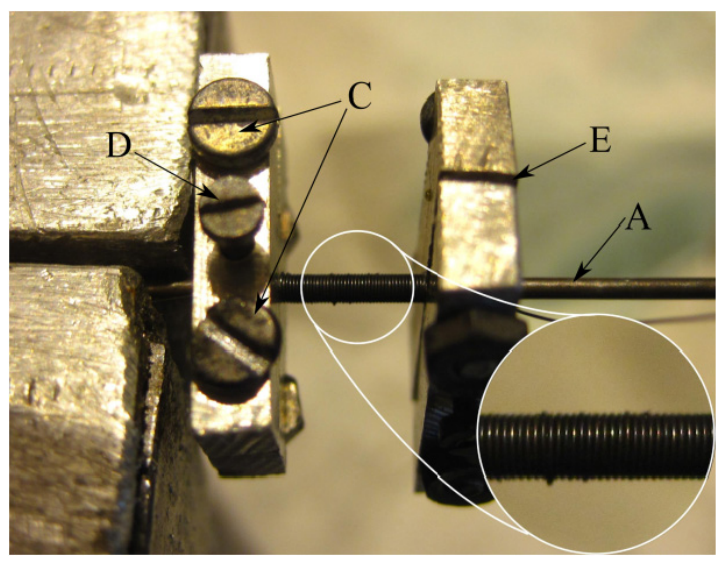

Figure 5. Fabrication method of the SMA springs. The wire is wound around a steel bar and fixed by two aluminum elements. Refer to figure 4 for the meaning of the letters.

wire diameter and spring index (ratio of average spring diameter and wire diameter). The springs built have a wire diameter of $0.2 \mathrm{~mm}$ and spring index equal to 6 , so the diameter of the core utilized for this specific case is $1 \mathrm{~mm}$.

This process avoids unwinding of the wire during the annealing process, when the activation temperature is reached and the material tends to regain the initial linear shape.

\subsection{Mechanical model validation}

The mechanical model was validated on springs with different geometrical characteristics. A total of 8 springs were tested: five of them were made with a $0.2 \mathrm{~mm}$ diameter wire, spring index equal to 6 , and 6 coils, and the remaining three were made with a $0.2 \mathrm{~mm}$ wire, spring index equal to 8.5 , and 15 coils. The springs were clamped in a material testing machine to measure the spring's resistant force while a tensile force was applied. Each test consisted in elongating the spring from the fully contracted position to maximum elongation, defined by the model. For each spring a minimum of three trials were performed to check repeatability too. For austenitic phase tests, a PWM control was used: a heating time of $1 \mathrm{~s}$ applying the current needed for a fast activation was used to activate the spring, then the duty cycle was adjusted to avoid overheating (which could damage and degrade spring performance). All the results are summarized in table 1 where a comparison between experimental results and model predictions are shown.

The mean error for the first set of springs, between the experimental measurements and the model, is around 5\% in the austenitic phase, while it is around $10 \%$ for the martensitic phase. In the second set the error is $3 \%$ in the austenitic phase and $5 \%$ in the martensitic phase.

Results on the repeatability of the spring performance obtained with the elongation tests are shown in figure 6 . The maximum force derived from the model, with the constraint of maximum internal stress fixed at $450 \mathrm{MPa}$, is $1.18 \mathrm{~N}$, and the maximum stroke is $246 \%$. The spring can exert the required force with a linear characteristic. The results are very 
Table 1. Mechanical model validation on eight springs. The spring rate is measured in the martensitic $\left(k_{c}\right)$ and austenitic $\left(k_{h}\right)$ phase and compared to the theoretical value.

\begin{tabular}{|c|c|c|c|c|}
\hline & \multicolumn{4}{|c|}{ Wire diameter: $0.2 \mathrm{~mm}$-spring index: 6 —coils: 6} \\
\hline & \multicolumn{2}{|c|}{ Austenite } & \multicolumn{2}{|c|}{ Martensite } \\
\hline & $\begin{array}{l}k_{h}-\text { model } \\
\left(\mathrm{N} \mathrm{mm}^{-1}\right)\end{array}$ & $\begin{array}{l}k_{h} \text {-experimental } \\
\left(\mathrm{N} \mathrm{mm}^{-1}\right)\end{array}$ & $\begin{array}{l}k_{c}-\text { model } \\
\left(\mathrm{N} \mathrm{mm}^{-1}\right)\end{array}$ & $\begin{array}{l}k_{c} \text {-experimental } \\
\left(\mathrm{N} \mathrm{mm}^{-1}\right)\end{array}$ \\
\hline Spring 1 & 0.39 & 0.33 & 0.16 & 0.13 \\
\hline Spring 2 & 0.39 & 0.37 & 0.16 & 0.15 \\
\hline Spring 3 & 0.39 & 0.38 & & \\
\hline Spring 4 & 0.39 & 0.39 & & \\
\hline \multirow[t]{2}{*}{ Spring 5} & 0.39 & 0.38 & & \\
\hline & \multicolumn{4}{|c|}{ Wire diameter: $0.2 \mathrm{~mm}$ —spring index: $8.5 —$ coils: 15} \\
\hline Spring 1 & 0.057 & 0.053 & 0.023 & 0.021 \\
\hline Spring 2 & 0.057 & 0.065 & 0.023 & 0.025 \\
\hline Spring 3 & 0.057 & 0.058 & 0.023 & 0.019 \\
\hline
\end{tabular}

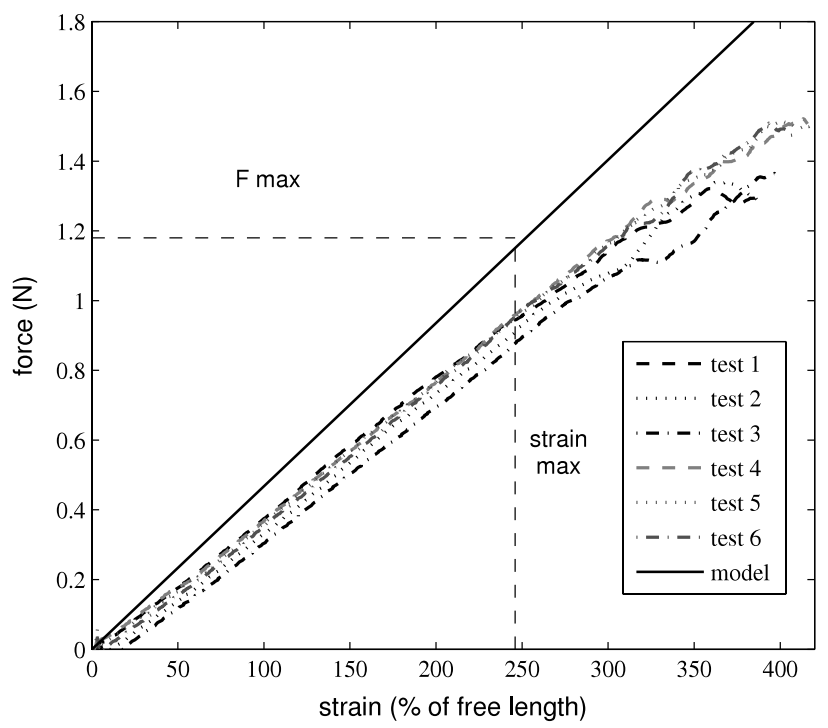

Figure 6. Six repeated elongation tests on the same spring in the austenitic phase. The force is measured and plotted as function of the percentage of elongation with respect to the spring at fully contracted length. Theoretical values are also shown for comparison purposes.

consistent with the model and show good repeatability, with very low errors, thus the model can be successfully used to design SMA springs from a mechanical point of view.

\subsection{Thermal model validation}

The aim of this part of the work was the application of the two methods for the measurement of the heat transfer coefficient, previously described, in order to make a comparison between the results obtained. The direct method was used to estimate the $h$ coefficient on a wire in air, as described in section 3.3. The experimental setup for the measurement of the temperature with the thermocouple led to an estimation of the coefficient of $128 \mathrm{~W} \mathrm{~m}^{-2} \mathrm{~K}^{-1}$. This coefficient was calculated by finding the curve, calculated by equation (5), that minimizes the mean quadratic error

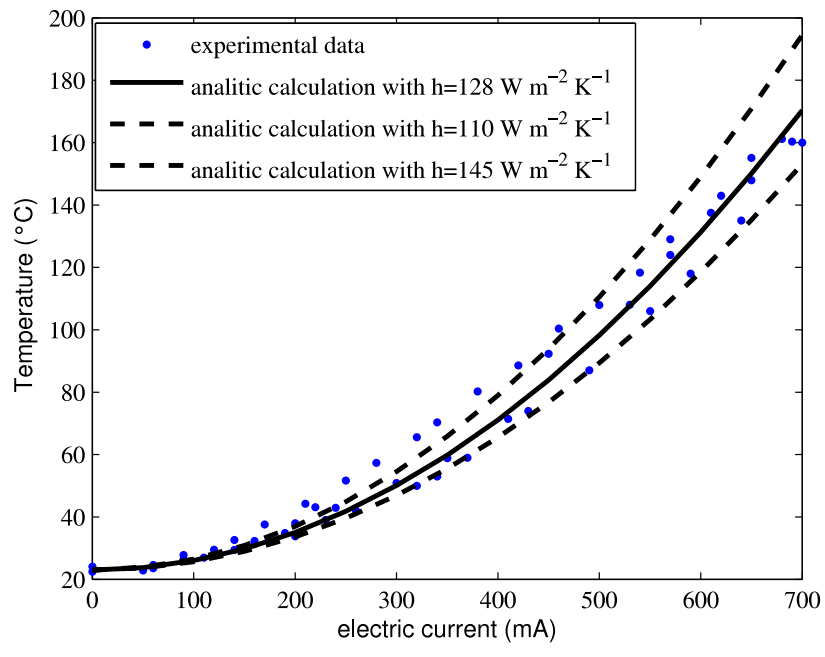

Figure 7. Steady state temperature reached for different supply currents (blue dots). Black line is the predicted temperature when the heat transfer coefficient $(h)$ is set to $128 \mathrm{~W} \mathrm{~m}^{-2} \mathrm{~K}^{-1}$ in the analytical calculation. Black dotted lines are the temperatures for a $\pm 15 \%$ difference $h$ values.

from the experimental data. Errors in this measurement are not negligible, and they are due to the low accuracy in measuring the activation temperatures and to the error in the measurements with the thermocouple. However a $15 \%$ error in the heat transfer coefficient has an acceptable impact on the estimated steady state temperature calculation, as shown in figure 7. For temperatures lower than $100{ }^{\circ} \mathrm{C}$, which is higher than the range of utilization of the alloy, an error of $15 \%$ from the nominal $h$ of $128 \mathrm{~W} \mathrm{~m}^{-2} \mathrm{~K}^{-1}$ gives a steady state temperature value that is still in the range of the experimental measurements and corresponds to a difference from the central value of around $10 \%$.

Another experimental setup was then realized to obtain the estimation of $h$ in the same environmental conditions by using the indirect method. Resistivity variation measures were performed on three wires with different lengths. The tested current ranges varied between 50 and $600 \mathrm{~mA}$ (figure 8). In all the three cases the maximum variation is visible for a current of $310 \mathrm{~mA}$. 


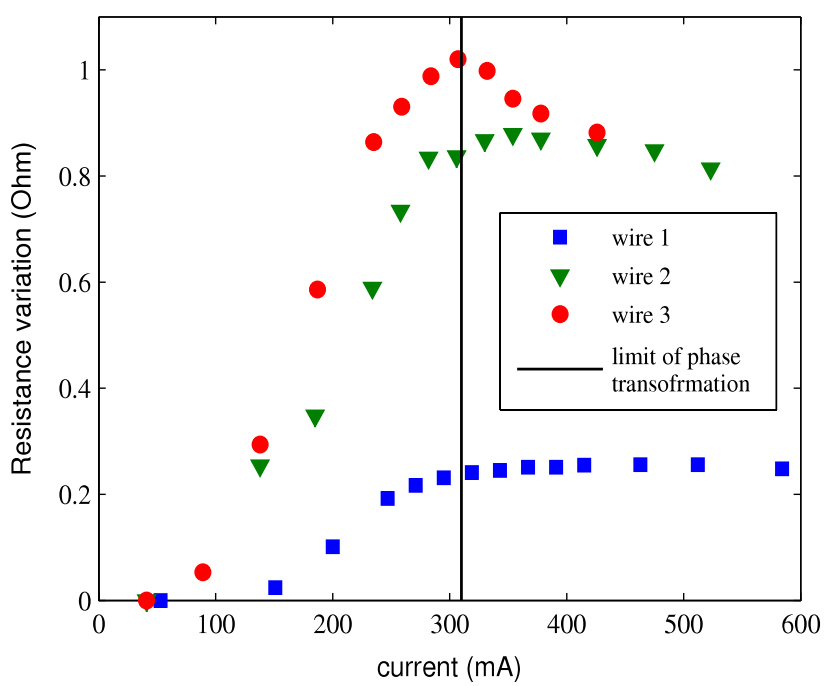

Figure 8. Resistance variation from the initial value and the steady state value for different supply currents in air. The maximum value is reached when the full austenitic phase is achieved. The resistance increases with temperature in the austenitic phase and overheating causes a reduction in the resistance variation.

Thus this value of current is considered as the value corresponding to a steady state temperature of $63^{\circ} \mathrm{C}$.

Data extrapolated from this experiment were used for the calculation of the heat transfer coefficient. $300 \mathrm{~mA}$ of current were imposed in equation (5) and the heat transfer coefficient found was $100 \mathrm{~W} \mathrm{~m}^{-2} \mathrm{~K}^{-1}$, differing from the theoretical value of $128 \mathrm{~W} \mathrm{~m}^{-2} \mathrm{~K}^{-1}$ by $20 \%$.

\section{Results and discussion}

\subsection{The effect of a liquid working environment}

The indirect method was then replicated for the wire in different ambient conditions. The first test was made in distilled water. Results show a peak in the initial and final resistance difference for $1600 \mathrm{~mA}$ (figure 9). This value leads to a heat transfer coefficient of nearly 2300 . Considerations on the error made in the experiment conducted in air are still valid also for this case. A $15 \%$ error in estimation of that coefficient introduces an error around $10 \%$ in the prediction of the steady state temperature in the range of temperatures around complete austenitic transformation $\left(63^{\circ} \mathrm{C}\right)$. The error increases with temperature.

Since the use of the wire in water is very power consuming, if compared to the use in air, some solutions are herein proposed to reduce the heat transfer coefficient. A first approach to the problem was by covering the wire with a commercially available $50 \mu \mathrm{m}$ thermo-restrictive PTFE sheath that provides thermal insulation during heating. The wire can be easily introduced in the sheath also when it is spring shaped. The thermal insulation capability of this solution was evaluated.

To quantify the benefits of introducing the sheath around the wire, the same set up (introduced in section 4.3) was used to measure the $h$ coefficient. Experimental results show an

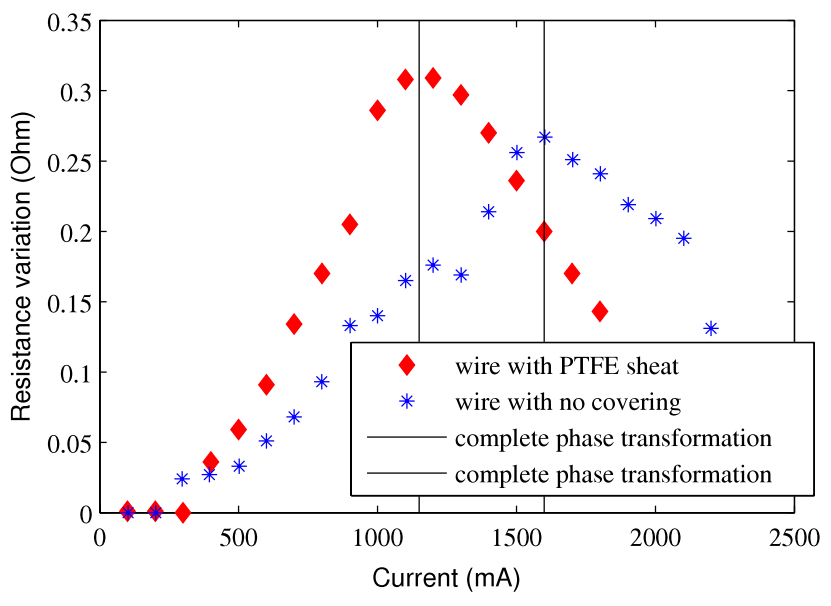

Figure 9. Resistance variation from the initial value and the steady state value for different supplied currents in water for a wire with a PTFE sheath.

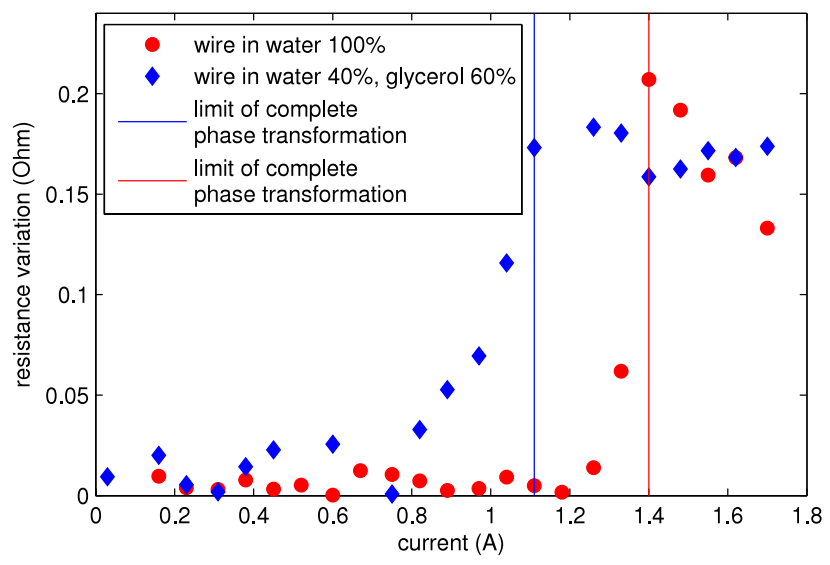

Figure 10. Comparison of resistance variation of wire immersed in $100 \%$ water (round red dots) and in a solution of $40 \%$ water and $60 \%$ glycerol (square blue dots).

activation current of $1150 \mathrm{~mA}$, corresponding to the peak in figure 9. An improvement in the heat exchange performance is evident; the heat transfer coefficient reduced from 2300 to $1200 \mathrm{~W} \mathrm{~m}^{-2} \mathrm{~K}^{-1}$, corresponding to a $47 \%$ reduction in electrical power needed to obtain the same temperature at steady state.

Further experiments were made on working environments with liquids that have a thermal conductivity lower than pure water, in order to reduce the heat transfer coefficient. The consequences on the bandwidth of the alloy are then examined, by simulating the thermodynamic response of the alloy.

Glycerol was chosen for its low thermal conductivity $\left(0.28 \mathrm{~W} \mathrm{~m}^{-1} \mathrm{~K}^{-1}\right.$ as compared to $0.6 \mathrm{~W} \mathrm{~m}^{-1} \mathrm{~K}^{-1}$ for water at $25^{\circ} \mathrm{C}$ ) and water solubility. Three solutions with different percentages of water were tested as environments for the actuator, repeating the same procedure for the estimation of heat transfer coefficient (figure 10). A decrease of the $h$ coefficient was reported from 2300 to $1280 \mathrm{~W} \mathrm{~m}^{-2} \mathrm{~K}^{-1}$. Results are reported in table 2. 
Table 2. Heat transfer coefficient measurement for a $0.2 \mathrm{~mm}$ wire in different environments and the relative power reduction to reach the same activation temperature.

\begin{tabular}{lllc}
\hline $\begin{array}{l}\text { Percentage of } \mathrm{H}_{2} \mathrm{O} \\
\text { in solution }(\%)\end{array}$ & $\begin{array}{l}\text { Peak } \\
\text { current }(A)\end{array}$ & $\begin{array}{l}\text { Heat transfer coeffi- } \\
\text { cient }\left(\mathrm{W} \mathrm{m}^{-2} \mathrm{~K}^{-1}\right)\end{array}$ & $\begin{array}{l}\text { Power reduction respect } \\
\text { to } 100 \% \mathrm{H}_{2} \mathrm{O}(\%)\end{array}$ \\
\hline 100 & 1.48 & 2279 & 0 \\
80 & 1.4 & 2040 & -11 \\
60 & 1.33 & 1841 & -19 \\
40 & 1.11 & 1282 & -44 \\
\hline
\end{tabular}

Table 3. Parameters of the austenitic phase used for the finite FEM model.

\begin{tabular}{ll}
\hline Thermal conductivity & $18 \mathrm{~W} \mathrm{~m}^{-1} \mathrm{~K}^{-1}$ \\
Specific heat & $320 \mathrm{~J} \mathrm{~kg}^{-1} \mathrm{~K}^{-1}$ \\
Resistivity & $76 \times 10^{-8} \Omega \mathrm{m}$ \\
Wire length & $0.02 \mathrm{~mm}$ \\
Wire diameter & $0.2 \mathrm{~mm}$ \\
\hline
\end{tabular}

A lower heat transfer coefficient, besides reducing power consumption, affects the heating and cooling time. Reducing the heating time is a desirable effect, while a long cooling time reduces the bandwidth of the actuator. A simple finite element model developed with the commercial software MSC MARC/MENTAT (Santa Ana, CA, USA) was used to determine the dynamic response of the temperature to an instantaneous variation of current in a wire. A wire with a $0.02 \mathrm{~mm}$ length and $0.2 \mathrm{~mm}$ diameter has a hexahedral mesh with 2380 elements. Data of the model are summarized in table 3 .

A current was imposed on one of the two faces of the cylinder, and a face film boundary condition was imposed on the surfaces where heat transfer occurs, with a determined $h$ coefficient for each simulation. Coefficients are derived from the previously described experiments. Activation in air, water and solutions of glycerol in water were simulated: a temperature of $68{ }^{\circ} \mathrm{C}$ was chosen as the target for all the tests, since it is above the temperature of complete austenitic transformation with no load. The heating time to target temperature in air is $6 \mathrm{~s}$, as well as the cooling time. A water environment reduces the time dramatically, even in the solution with glycerol: in an environment of $100 \%$ water, the heating and cooling times are both $0.5 \mathrm{~s}$; in a solution of $40 \%$ water and $60 \%$ glycerol an increase to $1 \mathrm{~s}$ is observed (figure 11). For the application in the bio-inspired octopus arm manipulator it is an acceptable threshold, which sets the maximum frequency for a complete heating-cooling cycle at $0.5 \mathrm{~Hz}$ in the case of minimum power consumption (environment $60 \%$ glycerol, $40 \%$ water), and at $1 \mathrm{~Hz}$ in the case of maximum power consumption (environment $100 \%$ water).

\subsection{Design process}

The workflow of the design process can be summarized in the following steps:

(1) Define the SMA properties, i.e., Young modulus of both phases and maximum internal stress allowed.

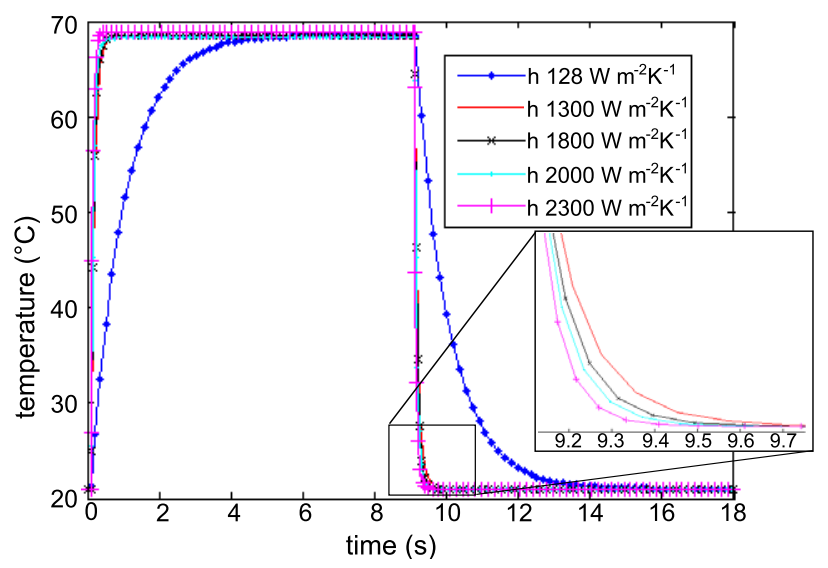

Figure 11. Simulated temperature variation in response to an instantaneous input current for $9 \mathrm{~s}$, for various ambient: air $\left(h=120 \mathrm{~W} \mathrm{~m}^{-2} \mathrm{~K}^{-1}\right), 100 \%$ water $\left(h=2300 \mathrm{~W} \mathrm{~m}^{-2} \mathrm{~K}^{-1}\right)$ and various solution of water and glycerol

$\left(h=1300-2000 \mathrm{~W} \mathrm{~m}^{-2} \mathrm{~K}^{-1}\right)$.

(2) Define the variable parameters range, i.e. wire diameter, spring diameter and number of coils.

(3) Solve the mechanical model for all combinations of variables.

(4) Select the springs that satisfy the requirements of force and strain.

(5) Among the solutions select the spring that optimizes one parameter (e.g. lower power consumption or highest force).

(6) Find the maximum operating force.

(7) Derive the phase transition temperature for activation.

(8) Calculate the power needed for activation, depending on the external environment.

These are the indications for the design process for the dimensioning of a single spring actuator; all the considerations are valid for both commercial springs or manually fabricated springs. The working environment influences the bandwidth and the power consumption for the activation, leaving unvaried the mechanical performances. All the steps were validated singularly on many spring prototypes, which are considered as general case studies.

\section{Conclusions}

The work presented here consists of the design and manufacturing process, as well as the thermodynamic 
characterization of a SMA spring, starting with the specifications of force required and the working space available for the actuator. This paper can be considered as a guide for the design of SMA spring actuators that can achieve the desired performance with a good accuracy. A mechanical model was first presented and validated, including the optimization protocol to find the configuration that best fits the requirements. A method for fabrication and the thermal treatment to obtain repeatable results is proposed. The model was validated with different springs, finding an error on the estimation of the spring rate of less than $10 \%$. A procedure for the measurement of the heat transfer coefficient is proposed, based on resistivity change of the alloy due to phase transition. This semi-empirical method was validated and an error of less than $20 \%$ on the final results was found. It was also demonstrated that it is possible to reduce this error by up to $10 \%$, considering the estimation of the temperature at steady state as the most important parameter for the spring design. A method to reduce the power consumption in a highly dissipative medium such as water is also proposed: covering the wire with a thin PTFE sheath reduces the power requirement by $47 \%$. A solution of glycerol and water instead of just water as working environment can reduce the power consumption by nearly $45 \%$. Better results in terms of electric power reduction could be obtained by combining the two solutions. The influence on the actuator bandwidth was investigated using a finite element model: the use of glycerol can increase the heating and cooling time from 0.5 to $1 \mathrm{~s}$. This method was successfully used to design and fabricate the actuators of the first prototype of an octopus-like robot arm.

\section{Acknowledgment}

This work was supported by the European Commission in the ICT-FET OCTOPUS Integrating Project, under contract No. 231608.

\section{References}

[1] Ikuta K 1990 Micro/miniature shape memory alloy actuator Proc. Int. Conf. on Robotics and Automation vol 3, pp 2156-61

[2] Mavroidis C 2002 Development of advanced actuators using shape memory alloys and electrorheological fluids Res. Nondestruct. Eval. 14 1-32

[3] Nespoli A, Besseghini S, Pittaccio S, Villa E and Viscuso S 2010 The high potential of shape memory alloys in developing miniature mechanical devices: a review on shape memory alloy mini-actuators Sensors Actuators A 158 149-60

[4] Wang Z, Hang G, Li J, Wang Y and Xiao K 2008 A micro-robot fish with embedded SMA wire actuated flexible biomimetic fin Sensors Actuators A 144 354-60

[5] Shinjo N and Swain G W 2004 Use of a shape memory alloy for the design of an oscillatory propulsion system IEEE $J$. Ocean. Eng. 29 750-5
[6] Garner L J, Wilson L N, Lagoudas D C and Rediniotis O K 2000 Development of a shape memory alloy actuated biomimetic vehicle Smart Mater. Struct. $9673-783$

[7] Zhang Y, Li S, Ma J and Yang J 2005 Development of an underwater oscillatory propulsion system using shape memory alloy Proc. Int. Conf on Mechatronics and Automation vol 4, pp 1878-83

[8] Cho K J, Koh J S, Kim S, Chu W S, Hong Y and Ahn S H 2009 Review of manufacturing processes for soft biomimetic robots Int. J. Precis. Eng. Manuf. 10 171-81

[9] Ballandras S, Calin M, Zissi S, Bertsch A, André J C and Hauden D 1997 Microstereophotolithography and shape memory alloy for the fabrication of miniaturized actuators Sensors Actuators A 62 741-7

[10] Bergamasco M, Salsedo F and Dario P 1989 A linear SMA motor as direct-drive robotic actuator Proc. Int. Conf. on Robotics and Automation vol 1, pp 618-23

[11] Wen M, Tu G F, Zong Q Y and Xie C X 1994 A study of NiTi shape memory alloy springs and its application in a new robotic actuator Proc. Int. Conf. on Industrial Technology pp 215-9

[12] Baohua H and Kai Y 2009 Research on novel compact actuators based on shape memory alloy springs Proc. Int. Conf. on Electrical Machines and Systems pp 1-4

[13] Guo S, Sun X, Ishii K and Guo J 2008 SMA actuator-based novel type of peristaltic micropump Proc. Int. Conf. on Information and Automation pp 1620-5

[14] Majima S, Kodama K and Hasegawa T 2001 Modeling of shape memory alloy actuator and tracking control system with the model IEEE Trans. Control Syst. Technol. 9 54-9

[15] Ikuta K, Tsukamoto M and Hirose S 1988 Shape memory alloy servo actuator system with electric resistance feedback and application for active endoscope Proc. Int. Conf. on Information and Automation vol 1, pp 427-30

[16] Auricchio F and Petrini L A 2004 Three-dimensional model describing stress-temperature induced solid phase transformations: solution algorithm and boundary value problems Int. J. Numer. Methods Eng. 61 807-36

[17] Brinson L C 1993 One-dimensional constitutive behavior of shape memory alloys: thermomechanical derivation with non-constant material functions J. Intell. Mater. Syst. Struct. $4229-42$

[18] Aguiar R, Savi M and Pacheco P 2010 Experimental and numerical investigations of shape memory alloy helical springs Smart Mater. Struct. 19025008

[19] Pathak A, Brei D and Luntz J 2010 Transformation strain based method for characterization of convective heat transfer from shape memory alloy wires Smart Mater. Struct. 19035005

[20] Bhattacharyya A, Sweeney L and Faulkner M G 2002 Experimental characterization of free convection during thermal phase transformations in shape memory alloy wires Smart Mater. Struct. 11411

[21] Cianchetti M, Follador M, Mazzolai B, Dario P and Laschi C 2012 Design and development of a soft robotic octopus arm exploiting embodied intelligence Proc. Int. Conf on Robotics and Automation (ICRA) vol 1, pp 5271-6

[22] Laschi C, Cianchetti M, Mazzolai B, Margheri L, Follador M and Dario P 2012 Soft Robot Arm Inspired by the Octopus Adv. Robot. 26 709-27

[23] Buehler W R 1963 Effects of low-temperature phase changes on the mechanical properties of alloys near composition TiNi J. Appl. Phys. 34 1475-7

[24] Patoor E, Lagoudas D C, Entchev P B, Brinson L C and Gao X 2006 Shape memory alloys, Part I: general properties and modeling of single crystals Mech. Mater. 38 391-429 